\title{
Predictive risk score model for severe fever with thrombocytopenia syndrome mortality based on qSOFA and SIRS scoring system
}

\author{
Li Wang ${ }^{*}$, Zhiqiang Zou, Kun Ding and Chunguo Hou
}

\begin{abstract}
Background: Severe fever with thrombocytopenia syndrome (SFTS) is a severe systemic virus infectious disease usually having multi-organ dysfunction which resembles sepsis.

Methods: Data of 321 patients with laboratory-confirmed SFTS from May 2013 to July 2017 were retrospectively analyzed. Demographic and clinical characteristics, calculated quick sequential organ failure assessment (qSOFA) score and systemic inflammatory response syndrome (SIRS) criteria for survivors and nonsurvivors were compared. Independent risk factors associated with in-hospital mortality were obtained using multivariable logistic regression analysis. Risk score models containing different risk factors for mortality in stratified patients were established whose predictive values were evaluated using the area under ROC curve (AUC).

Results: Of 321 patients, 87 died $(27.1 \%)$. Age $(p<0.001)$ and percentage numbers of patients with qSOFA $\geq 2$ and SIRS $\geq 2(p<0.0001)$ were profoundly greater in nonsurvivors than in survivors. Age, qSOFA score, SIRS score and aspartate aminotransferase (AST) were independent risk factors for mortality for all patients. qSOFA score was the only common risk factor in all patients, those age $\geq 60$ years and those enrolled in the intensive care unit (ICU). A risk score model containing all these risk factors (Model1) has high predictive value for in-hospital mortality in these three groups with AUCs (95\% Cl): 0.919 (0.883-0.946), 0.929 (0.862-0.944) and 0.815 (0.710-0.894), respectively. A model only including age and qSOFA also has high predictive value for mortality in these groups with AUCs (95\% Cl): 0.872 (0.830-0.906), 0.885(0.801-0.900) and 0.865 (0.767-0.932), respectively.
\end{abstract}

Conclusions: Risk models containing qSOFA have high predictive validity for SFTS mortality.

Keywords: Severe fever with thrombocytopenia syndrome, Risk score model, qSOFA, SIRS

\section{Background}

Severe fever with thrombocytopenia syndrome (SFTS) is an emerging hemorrhagic disease with high mortality of $12-30 \%$, which is caused by the SFTS virus (SFTSV) infection [1]. The clinical manifestations typically became worse within a week of admission, and most of them had multi-organ dysfunction. Previous studies have

\footnotetext{
*Correspondence: liliwang2200@163.com

Infectious disease department, Qishan (Infectious Disease) Hospital of Yantai, 62 Huanshan Road, Zhifu District, Yantai, Shandong 264001, People's Republic of China
}

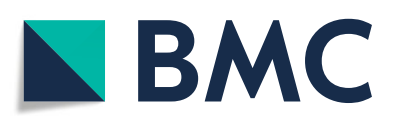

(c) The Author(s). 2020 Open Access This article is licensed under a Creative Commons Attribution 4.0 International License, which permits use, sharing, adaptation, distribution and reproduction in any medium or format, as long as you give appropriate credit to the original author(s) and the source, provide a link to the Creative Commons licence, and indicate if changes were made. The images or other third party material in this article are included in the article's Creative Commons licence, unless indicated otherwise in a credit line to the material. If material is not included in the article's Creative Commons licence and your intended use is not permitted by statutory regulation or exceeds the permitted use, you will need to obtain permission directly from the copyright holder. To view a copy of this licence, visit http://creativecommons.org/licenses/by/4.0/ The Creative Commons Public Domain Dedication waiver (http://creativecommons.org/publicdomain/zero/1.0/) applies to the data made available in this article, unless otherwise stated in a credit line to the data. shown many laboratory variables and clinical parameters to be associated with death including elevated levels of aspartate aminotransferase (AST), serum creatinine $(\mathrm{sCr})$, blood urea nitrogen (BUN), viral loads, age and neurological symptoms [2, 3]. Study has demonstrated that the inflammatory cytokine storm was associated with the severity of SFTS [4] and specific treatment for SFTS was not proved [5]. Limited data showed that ribavirin therapy was effective only in patients with a viral load below $1 \times 106$ copies/mL [2]. Therefore, the initial prediction of an adverse outcome is of utmost 
importance for taking effective preventative and combined internal medical therapeutic measures to prevent the disease from becoming worse which can be attained by monitoring clinical and laboratory variables.

Sepsis is a life-threatening organ dysfunction caused by the dysregulated host response to infection. Sequential organ failure assessment (SOFA), quick SOFA (qSOFA) scoring systems, and systemic inflammatory response syndrome (SIRS) criteria are usually used to evaluate the severity of sepsis [6]. Though the term sepsis may not be appropriate for life-threatening acute organ dysfunction caused by nonbacterial infections, such as SFTS, some authors have suggested using the term sepsis or analogous severe infectious course in this case [7]. SOFA, qSOFA scoring systems and SIRS criteria have widely applied for the prediction of in-hospital mortality who are likely to have sepsis (or analogous severe infectious course) in the intensive care unit (ICU) [6, 8]. However, their roles have not been well-evaluated for the prediction of in-hospital mortality of SFTS patients. Though several risk models have been established for the prediction of SFTS mortality, the indices included can not be available quickly after admission rendering delay of their utility, such as viral loads $[3,9]$. Compared to SOFA score of which many components were quickly unavailable in most patients, qSOFA score and SIRS criteria were composed of simple and easily obtained parameters [6]. qSOFA was defined as a score composed of three binary variables (tachypnoea, hypotension, and altered mental status) [10] and parameters included in SIRS criteria were respiratory rate, temperature, pulse and white blood cell count [6]. These variables can be obtained readily and promptly after patients on admission.

The purpose of this study was to evaluate the predictive validity of the qSOFA and SIRS score and the established risk models containing these two parameters at different clinical settings for in-hospital mortality of SFTS patients. Meanwhile, other inflammatory parameters including white blood count (WBC) and highsensitivity C-reactive protein (hs-CRP) were also analyzed for comparison.

\section{Methods}

\section{Patients and calculation of qSOFA and SIRS score}

Three hundred and twenty-one SFTS patients who were admitted to our hospital from May 2013 to July 2017 were included. Defined diagnosis of SFTS was made by detected positive SFTSV from peripheral blood samples using reverse transcription-polymerase chain reaction (RT-PCR). The following data were extracted for each patient after presentation: demographics and components of the SIRS criteria and qSOFA score (most within the first $24 \mathrm{~h}$ of admission). The qSOFA score includes respiratory rate $\geq 22$ / min, systolic blood pressure $\leq 100 \mathrm{mmHg}$, and abnormal mental status. SIRS criteria include respiratory rate $>20 / \mathrm{min}$; temperature $>38^{\circ} \mathrm{C}$ or $<36^{\circ} \mathrm{C}$; pulse > 90 beats/min; and white blood cell count $>12,000 / \mu \mathrm{L}$ or $<4000 / \mu \mathrm{L}$ [6]. Distribution of different qSOFA and SIRS score of all patients, survivors and nonsurvivors

Table 1 Demographics and baseline biochemical parameters, qSOFA and SIRS scores in survivors and nonsurvivors [median (IQR) or $(\bar{x} \pm$ SD)] of SFTS patients

\begin{tabular}{|c|c|c|c|c|}
\hline Parameters & All patients & Survivors & nonsurvivors & $P$ value \\
\hline $\mathrm{N},(\%)$ & $321(100)$ & $234(72.9)$ & $87(27.1)$ & $<0.001$ \\
\hline Age (year), mean $\pm S D$ & $63.8 \pm 11.2$ & $61.3 \pm 10.8$ & $70.6 \pm 9.2$ & $<0.0001$ \\
\hline Age <60, n (\%) & $111(34.6)$ & $97(41.5)$ & $14(16.1)$ & $<0.001$ \\
\hline Age $\geq 60, n(\%)$ & $210(65.4)$ & $137(58.6)$ & $73(83.9)$ & $<0.0001$ \\
\hline Male, n (\%) & $170(53)$ & 116(49.6) & $54(62.1)$ & $<0.05$ \\
\hline qSOFA score $\geq 2, \mathrm{n}(\%)$ & $44(13.7)$ & $8(3.4)$ & $36(41.4)$ & $<0.0001$ \\
\hline SIRS score $\geq 2, n(\%)$ & 124(38.6) & $66(28.2)$ & $58(66.7)$ & $<0.0001$ \\
\hline PCT $\geq 0.05, n(\%)$ & $114 / 253(44.9)$ & $91 / 185(49.2)$ & $54 / 68(79.9)$ & $<0.0001$ \\
\hline $0.5<\mathrm{PCT}<2, \mathrm{n}(\%)$ & $50(19.8)$ & $8(4.3)$ & $42(61.8)$ & $<0.0001$ \\
\hline$P C T \geq 2, n(\%)$ & $19(7.5)$ & $7(3.8)$ & $12(17.7)$ & $<0.0001$ \\
\hline Time in-hospital (days) (IQR) & $11(5-15)$ & $15(11-18)$ & $4(3-5)$ & $<0.0001$ \\
\hline plasma lactate (mmol/L) (IQR) & $1.2(1-1.78)$ & $1.1(0.9-1.4)$ & $1.7(1.23-2.78)$ & $<0.0001$ \\
\hline $\mathrm{WBC}\left(\times 10^{9} / \mathrm{L}\right)(\mathrm{IQR})$ & $3.15(1.9-5.0)$ & $3.1(1.9-4.3)$ & $3.5(1.8-6.6)$ & $>0.05$ \\
\hline AST (U/L) (IQR) & 183(86.6-365.5) & 155(83-326.5) & $428.6(170.6-1019)$ & $<0.0001$ \\
\hline $\mathrm{sCr}(\mu \mathrm{mol} / \mathrm{L})(\mathrm{IQR})$ & $65.8(57.6-81.0)$ & $65.1(54.2-74.4)$ & $79.4(65.7-126.2)$ & $<0.0001$ \\
\hline $\mathrm{Hs}-\mathrm{CRP}(\mathrm{mmol} / \mathrm{L})(\mathrm{IQR})$ & $3.8(1.44-11.4)$ & $1.8(1.26-5.7)$ & $0.36(0.11-1.02)$ & $<0.0001$ \\
\hline
\end{tabular}

qSOFA quick sequential organ failure assessment, SIRS systemic inflammatory response syndrome, $P C T$ Procalcitonin, WBC white blood cell, $A S T$ aspartate aminotransferase, $s C r$ serum creatinine, $h s-C R P$ high-sensitivity $C$-reactive protein 
was measured and qSOFA score and SIRS score $\geq 2$ was regarded as high. The predictive risk models for prognosis in several subgroups were constructed based on regression coefficients of the independent risk factors of outcome obtained from multi-factorial logistic regression analysis.

This study was conducted according to the Helsinki II Declaration and was approved by the ethics committee of Infectious disease hospital of Yantai. Written informed consent was obtained from the patients.

\section{Statistical analysis}

Data are presented as numbers (percentages) and mean with standard deviation. The student $t$ test or MannWhitney $U$ test was used for the comparison of variables with normal or abnormal distribution between the survival and nonsurvival groups. Proportions of variables between groups were compared using $\chi^{2}$ test. Independent risk factors associated with mortality were derived using multivariate logistic regression analysis. Variables that have been demonstrated as independent risk factors in our previous study [3] and those inflammatory indices such as
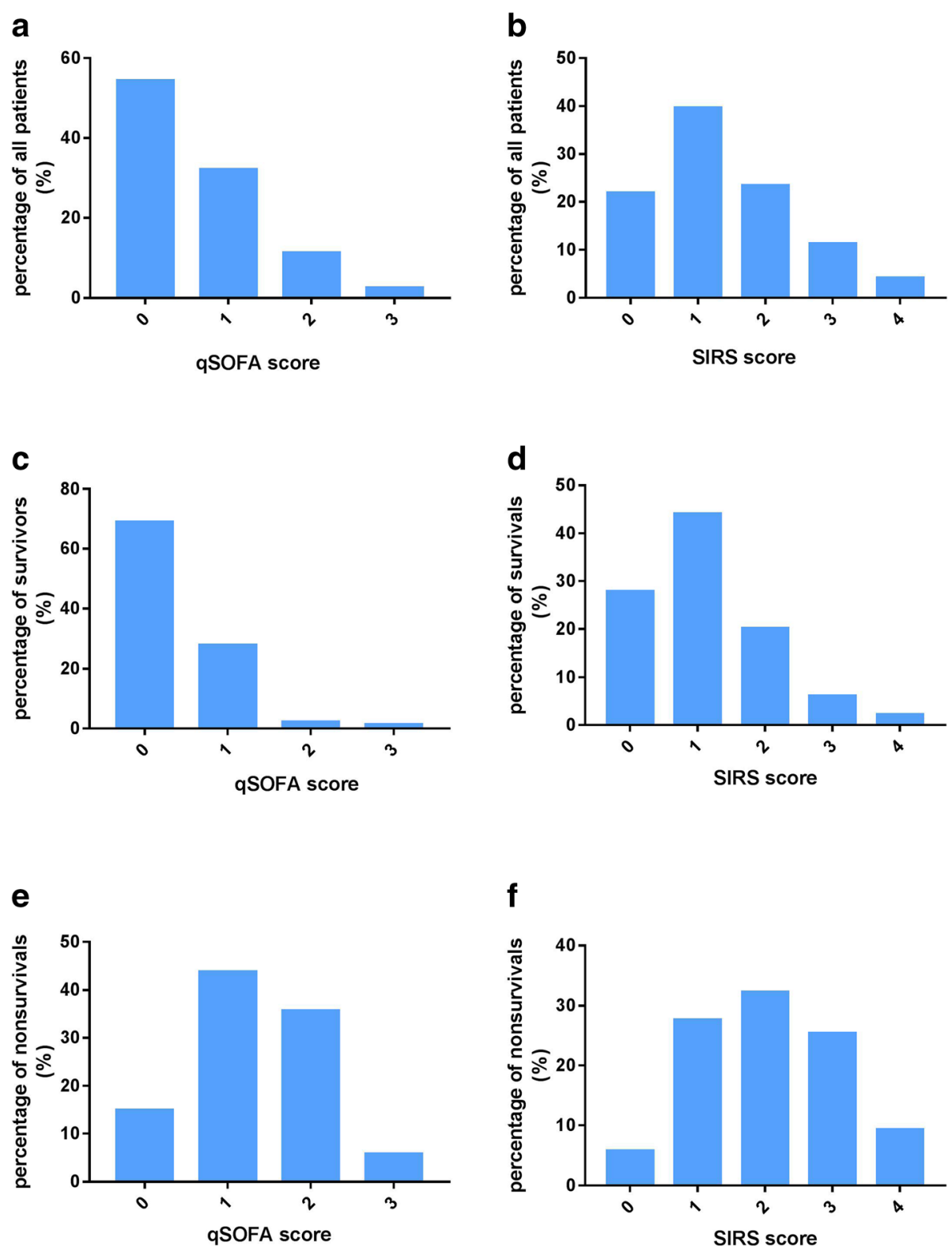

Fig. 1 Patients number percentage with different qSOFA scores and SIRS scores. (a) Number distribution with qSOFA scores in all patients. (b) Number distribution with SIRS scores in all patients. (c) Number distribution of qSOFA scores in survivors. (d) Patients number distribution with SIRS scores in survivors. (e) Patients number distribution of qSOFA scores in nonsurvivors. $\mathbf{f}$ Patients number distribution of SIRS scores in nonsurvivors 
WBC and hs-CRP, and qSOFA and SIRS score were included in a multivariate logistic regression model. Risk score models based on independent risk factors at different clinical settings were developed to reflect the underlying risk of a patient developing a fatal outcome. The discrimination of the predictive power of the independent risk factors and models were evaluated using the area under the receiver operating characteristic curve (AUC). Sensitivity (SEN), specificity (SPE), negative predictive value (NPV), positive predictive value (PPV) positive likelihood ratio $(\mathrm{LR}+)$ and negative likelihood ratio (LR-) were obtained using MedCalc software for each score. Cutoff values were chosen with the highest Youden index and the optimal ones the software provided. The KaplanMeier survival analysis was utilized to compare the cumulative risk for death in high-risk and low-risk groups according to cutoff values of the model score obtained from ROC analysis, and the significance of difference was tested with the log-rank test. Analyses were conducted using the SPSS, version 23.0, software (IBM, Armonk, NY, USA). $p$ values $<0.05$ were considered significant.

\section{Results}

Demographics and baseline qSOFA and SIRS scores in survivals and nonsurvivals

Of 370 suspected SFTS patients who were admitted to our hospital from May 2013 to July 2017, 321 were confirmed by detected positive SFTSV and were included in this study. Mean age of all included patients was $63.8 \pm$
11.2 years. Eighty seven patients (27.1\%) died during hospitalization who were older than those who survived (70.6 \pm 9.2 vs $61.3 \pm 10.8$ years, $p<0.0001)$. Percentage of male patients in nonsurvivors was greater than in survivors $(p<0.05)$. Proportions of patients with qSOFA and SIRS score $>2$ were dramatically increased in nonsurvivors compared with survivors $(p<0.0001)$. Hospital stays and biochemical parameters had significant differences between the two groups. Data are shown in Table 1 and Fig. 1.

\section{Predictive values of independent risk factors, and established risk score models for in-hospital mortality of SFTS patients \\ Predictive values of independent risk factors and risk models for all patients}

Multivariate logistic regression analysis showed that age, AST, qSOFA and SIRS scores were the independent risk factors for in-hospital mortality of all SFTS patients. AUCs $(95 \%$ CI), cutoff values, SEN, SPE, PPV and NPV of these factors for the prediction of in-hospital mortality are included in Table 2. Based on values and regression coefficient of these risk factors, a risk score model was constructed as $\mathrm{M} 1=0.102 \times$ age $+0.002 \times \mathrm{AST}+$ $1.296 \times$ qSOFA score $+0.486 \times$ SIRS score. AUC $(95 \% \mathrm{CI})$ of M1 was $0.919(0.883-0.946)$ with odd ratio (OR) (95\% $\mathrm{CI})=2.95(2.308-3.771)$ at the cutoff value of 9.22 (Table 2, Fig. 2a). Kaplan-Meier survival analysis showed

Table 2 Predictive values of independent risk factors and established models containing different risk factors for in-hospital mortality of all SFTS patients

\begin{tabular}{|c|c|c|c|c|c|c|c|c|c|c|}
\hline Parameters & OR (95\%) & $P$ value & Cutoff value & AUC $(95 \%$ & SEN & SPE & PPV & NPV & LR+ & LR- \\
\hline \multirow[t]{2}{*}{ Age (years) } & 1.107 (1.039-1.176) & $<0.0001$ & $>66$ & $0.742(0.69-0.789)$ & 70.1 & 68.4 & 45.2 & 86 & 2.22 & 0.44 \\
\hline & & & $>74$ & & 39.1 & 89.3 & 57.6 & 79.8 & 3.66 & 0.68 \\
\hline \multirow[t]{2}{*}{ AST (U/L) } & $1.002(1.000-1.003)$ & 0.01 & $>205$ & $0.794(0.729-0.849)$ & 77.4 & 67 & 45.8 & 89.1 & 2.34 & 0.34 \\
\hline & & & $>735$ & & 38.1 & 96.1 & 78 & 81.2 & 9.86 & 0.64 \\
\hline \multirow[t]{2}{*}{ qSOFA } & 3.654 (1.488-6.378) & $<0.0001$ & $>0$ & $0.818(0.774-0.858)$ & 85.1 & 68.8 & 50.3 & 95.2 & 2.73 & 0.22 \\
\hline & & & $>1$ & & 41.4 & 95.6 & 81.8 & 81.6 & 12.1 & 0.61 \\
\hline \multirow[t]{2}{*}{ SIRS } & $1.625(1.025-2.581)$ & 0.039 & $>1$ & $0.740(0.688-0.787)$ & 66.7 & 71.8 & 46.8 & 81.3 & 2.36 & 0.46 \\
\hline & & & $>2$ & & 34.5 & 91.9 & 61.2 & 79 & 4.25 & 0.71 \\
\hline \multirow[t]{2}{*}{ M1 } & $2.95(2.308-3.771)$ & $<0.0001$ & $>9.22$ & $0.919(0.883-0.946)$ & 81.6 & 85.9 & 68.3 & 92.6 & 5.79 & 0.21 \\
\hline & & & $>10.27$ & & 65.2 & 95.7 & 85.1 & 88.2 & 15.3 & 0.36 \\
\hline \multirow[t]{2}{*}{ M2 } & $3.122(2.401-4.033)$ & $<0.0001$ & $>8.034$ & $0.894(0.855-0.925)$ & 87.4 & 75.2 & 56.7 & 94.1 & 3.52 & 0.17 \\
\hline & & & $>9.942$ & & 51.7 & 91.9 & 90 & 84.5 & 24.2 & 0.349 \\
\hline \multirow[t]{2}{*}{ M3 } & $2.986(2.324-3.837)$ & $<0.0001$ & $>8.25$ & $0.899(0.861-0.930)$ & 85.1 & 78.6 & 59.7 & 93.4 & 3.98 & 0.19 \\
\hline & & & $>9.06$ & & 71.3 & 91.5 & 75.6 & 89.5 & 8.34 & 0.31 \\
\hline \multirow[t]{2}{*}{ M4 } & $3.26(2.437-4.297)$ & $<0.0001$ & $>8.034$ & $0.872(0.830-0.906)$ & 85.9 & 81.6 & 60.6 & 90.1 & 4.13 & 0.3 \\
\hline & & & $>8.44$ & & 63.2 & 91.0 & 72.4 & 86.9 & 7.04 & 0.4 \\
\hline
\end{tabular}

OR odd ratio, $C I$ confidence interval, $A U C$ area under ROC curve, $A S T$ aspartate aminotransferase, qSOFA quick sequential organ failure assessment, SIRS systemic inflammatory response syndrome, SEN sensitivity, SPE specificity, $P P V$ positive predictive value, NPV negative predictive value, $L R+$ positive likelihood ratio, $L R$ negative likelihood ratio 


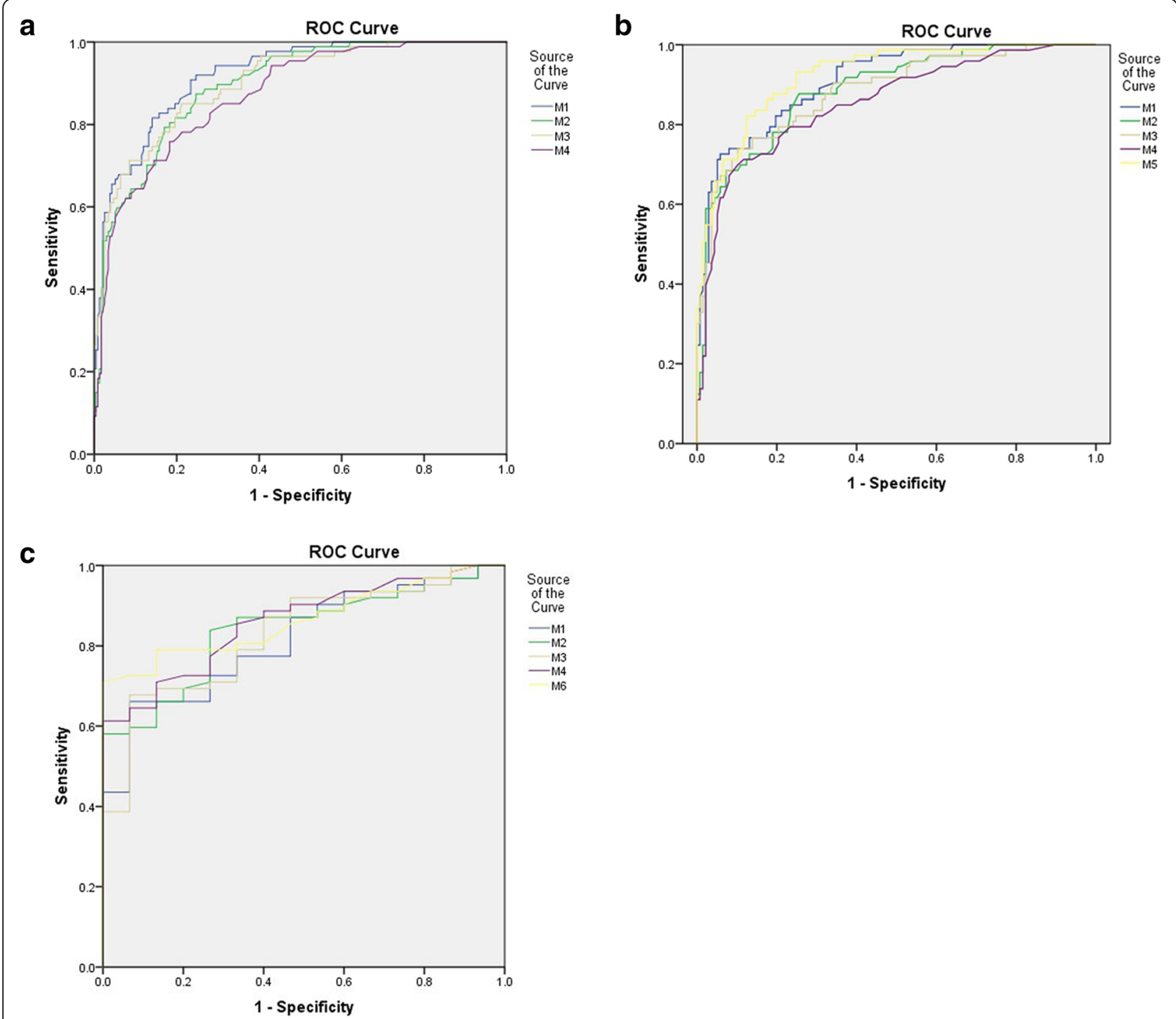

Fig. 2 (a) AUCs of M1, M2, M3 and M4 for in-hospital mortality in all patients. (b) AUCs of M1, M2, M3, M4 and M5 for for in-hospital mortality for patients at age $\geq 60$ years. (c) AUCs of M1, M2, M3, M4 and M6 for for in-hospital mortality for patients enrolled in ICU

a strong difference between high-risk and low-risk groups (log-rank test, $\chi^{2}=1551.1, p<0.0001$ ) (Fig. 3a).

Considering relative small regression coefficient of AST and SIRS scores, we modified the models into simpler ones as:

$$
\begin{aligned}
\text { M2 }(\text { model } 2)= & 0.102 \times \text { age }+1.296 \times \text { qSOFA } \\
& +0.486 \times \text { SIRS } \\
\text { M3 }(\text { model } 3)= & 0.102 \times \text { age }+0.002 \times \text { AST }+1.296 \\
& \times \text { qSOFA } \\
\text { M4 }(\text { model } 4)= & 0.102 \times \text { age }+1.296 \times \text { qSOFA }
\end{aligned}
$$

These three models had comparable predictive power and had a relative less power than M1 (Table 2, Fig. 2).
Survival analysis demonstrated a strong statistical significance between high and low-risk groups of M1 and M4 (selection based on the highest AUC and OR values) $(p<0.0001)$ (Fig. 3a, b).

\section{Predictive values of independent risk factors and risk models for patients with age $\geq 60$ years}

The four models also had high predictive values for inhospital mortality in those patients at age $\geq 60$ years (Table 3). Logistic regression analysis indicated that age, plasma lactate, serum AST and hs-CRP levels, and qSOFA and SIRS scores were the independent risk factors for in-hospital mortality in this group of patients. Based on these factors, we established another risk score model: 

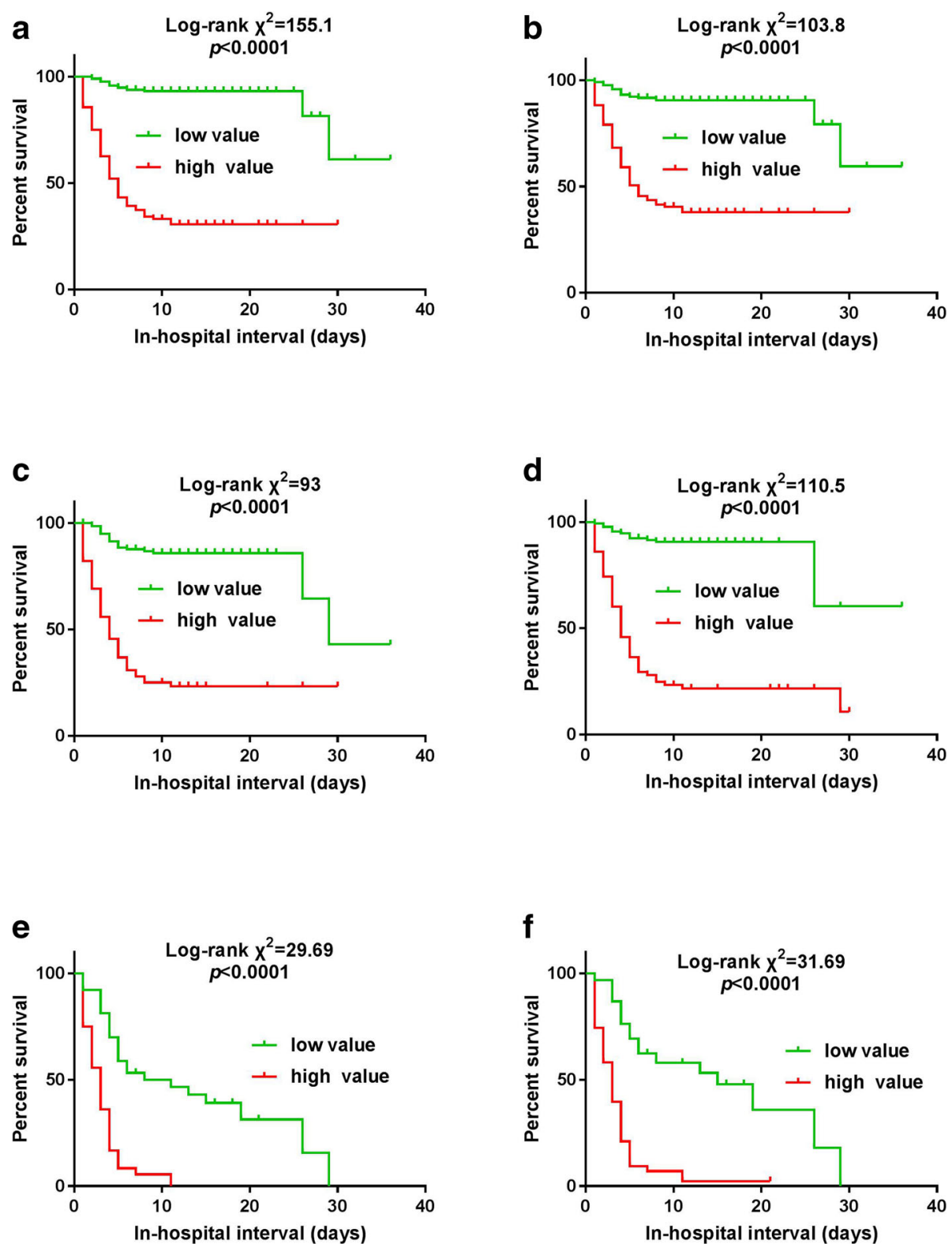

Fig. 3 (a) Survival curve for all patients at cutoff value of $>9.22$ of M1. (b) Survival curve for all patients at cutoff value of $>8.034$ of M4. (c) Survival curve for patients of $\geq 60$ years at cutoff value of $>8.64$ of M4. (d) Survival curve for patients of $\geq 60$ years at cutoff value of $>13.68$ of M5. (e) Survival curve for patients enrolled in ICU at cutoff value of $>8.57$ of M4. (f) Survival curve for patients enrolled in ICU at cutoff value of $>6.42$ of M6

$$
\begin{aligned}
\mathrm{M} 5= & 0.127 \times \text { age }+0.953 \times \text { lactate }+0.002 \times \mathrm{AST} \\
& +0.056 \times \mathrm{Hs}-\mathrm{CRP}+0.86 \times \mathrm{qSOFA}+0.866 \\
& \times \text { SIRS }
\end{aligned}
$$

This model (M5) has the highest predictive value among these models for in-hospital mortality in this group of patients (Table 3, Fig. 2b).

Survival analysis manifested a profound statistical significance between high and low-risk groups of M4 and
M5 (selection based on the highest AUC and OR values) $p<0.0001$ ) (Fig. 3c, d).

\section{Predictive values of independent risk factors and risk models for patients in ICU}

The former four models also have high predictive values for in-hospital mortality in those patients enrolled in ICU at admission or transferred to (Table 4, Fig. 2c).

Univariate logistic regression showed that age and qSOFA score were the independent risk factors for mortality of this subgroup of patients. Multivariate logistic 
Table 3 Predictive values of independent risk factors and established models containing different risk factors for in-hospital mortality of patients with age $\geq 60$ years

\begin{tabular}{|c|c|c|c|c|c|c|c|c|c|c|}
\hline Parameters & OR $(95 \% \mathrm{Cl})$ & $P$ value & Cutoff value & AUC (95\% Cl) & SEN & SPE & PPV & NPV & LR+ & LR- \\
\hline \multirow[t]{2}{*}{ Age (years) } & $1.135(1.037-1.243)$ & 0.006 & $>72$ & $0.706(0.640-0.767)$ & 56.2 & 74.5 & 53.9 & 76.1 & 2.2 & 0.59 \\
\hline & & & $>74$ & & 46.6 & 81.8 & 57.6 & 74.2 & 2.55 & 0.65 \\
\hline \multirow[t]{2}{*}{ lactate (mmol/L) } & $2.592(1.06-6.34)$ & 0.037 & $>1.17$ & $0.757(0.674-0.828)$ & 85.5 & 52 & 56.6 & 83 & 1.78 & 0.28 \\
\hline & & & $>2.1$ & & 38.2 & 94.7 & 84 & 67.6 & 7.16 & 0.65 \\
\hline \multirow[t]{2}{*}{ Hs-CRP (mmol/L) } & $1.058(1.007-1.11)$ & 0.024 & $>6.3$ & $0.729(0.69-0.789)$ & 81.7 & 54.7 & 48.3 & 85.2 & 1.81 & 0.33 \\
\hline & & & $>3.14$ & & 25.4 & 95.6 & 75 & 71.2 & 5.79 & 0.78 \\
\hline \multirow[t]{2}{*}{ AST (U/L) } & $1.002(1.000-1.003)$ & 0.01 & $>203$ & $0.779(0.717-0.834)$ & 78.8 & 66.4 & 54.9 & 85.8 & 2.35 & 0.32 \\
\hline & & & $>722$ & & 39.4 & 95.6 & 82.4 & 75.3 & 9 & 0.63 \\
\hline \multirow[t]{2}{*}{ qSOFA } & $2.364(1.142-4.89)$ & 0.02 & $>0$ & $0.818(0.759-0.867)$ & 83.6 & 71.5 & 61 & 89.1 & 2.94 & 0.23 \\
\hline & & & $>1$ & & 39.7 & 97.1 & 87.9 & 75.1 & 13.6 & 0.62 \\
\hline \multirow[t]{2}{*}{ SIRS } & $2.377(1.025-2.581)$ & 0.008 & $>1$ & $0.778(0.716-0.833)$ & 68.5 & 76.6 & 61 & 82 & 2.93 & 0.41 \\
\hline & & & $>2$ & & 35.6 & 94.9 & 78 & 73.4 & 6.97 & 0.68 \\
\hline M1 & $3.144(2.319-4.263)$ & $<0.0001$ & $>10.272$ & $0.909(0.862-0.944)$ & 72.6 & 94.2 & 86.9 & 86.6 & 12.43 & 0.29 \\
\hline \multirow[t]{2}{*}{ M2 } & $3.508(2.506-4.910)$ & $<0.0001$ & $>8.544$ & $0.886(0.835-0.926)$ & 87.7 & 75.5 & 64.6 & 91.9 & 3.43 & 0.17 \\
\hline & & & $>9.942$ & & 58.9 & 97.8 & 26.9 & 0.42 & 26.9 & 0.42 \\
\hline M3 & $3.11(2.286-4.231)$ & $<0.0001$ & $>9.3$ & $0.883(0.831-0.923)$ & 73.97 & 89.78 & 79.4 & 86.6 & 7.24 & 0.29 \\
\hline M4 & $3.533(2.494-5.005)$ & $<0.0001$ & $>8.64$ & $0.885(0.801-0.900)$ & 69.86 & 89.78 & 78.5 & 84.8 & 6.84 & 0.3 \\
\hline M5 & $2.508(1.951-3.223)$ & $<0.0001$ & $>13.68$ & $0.924(0.879-0.956)$ & 82.2 & 87.6 & 77.9 & 90.2 & 6.62 & 0.2 \\
\hline
\end{tabular}

$O R$ odd ratio, $C l$ confidence interval, $A U C$ area under ROC curve, $A S T$ aspartate aminotransferase, $h s$-CRP high-sensitivity C-reactive protein, $q S O F A$ quick sequential organ failure assessment, SIRS systemic inflammatory response syndrome, SEN sensitivity, SPE specificity, PPV positive predictive value, NPV negative predictive value, $L R+$ positive likelihood ratio, $L R$ - negative likelihood ratio

regression analysis demonstrated that qSOFA score was the only independent risk factor for mortality of these patients. Based on these two parameters we built another model that has the same index of M4 with different regression coefficient:

$$
\text { M6 }(\text { model } 6)=0.071 \times \text { age }+1.877 \times \text { qSOFA }
$$

This model has the highest predictive value among these models for in-hospital mortality in this subgroup of patients (Table 4, Fig. 2c).
Survival analysis showed a significant statistical difference between high and low-risk groups of M4 and M6 (selection based on the highest AUC and OR values) $p<$ 0.0001) (Fig. 3e, f).

Of note, no multi-colinearity between SOFA and SIRS were detected in three models cotaining these two parameters.

\section{Discussion}

SFTS is a severe emerging virus infectious disease which is a great life-threat to people locally and globally. In this study, we evaluated the varied predictive validity of

Table 4 Predictive values of independent risk factors and established models containing different risk factors for in-hospital mortality of patients enrolled in intensive care unit (ICU)

\begin{tabular}{|c|c|c|c|c|c|c|c|c|c|c|}
\hline Parameters & OR (95\% Cl) & $P$ value & Cutoff value & AUC (95\% Cl) & SEN & SPE & PPV & NPV & LR+ & LR- \\
\hline age (years) & $1.073(1.008-1.142)$ & 0.028 & $>68$ & $0.685(0.570-0.787)$ & 62.9 & 73.3 & 90.7 & 32.4 & 2.36 & $\overline{0.51}$ \\
\hline qSOFA & 6.299 (1.932-20.53) & 0.02 & $>0$ & $0.828(0.726-0.905)$ & 79.03 & 73.3 & 92.5 & 45.8 & 2.96 & 0.29 \\
\hline \multirow[t]{2}{*}{ M1 } & $1.875(1.287-2.732)$ & 0.001 & $>10.15$ & $0.815(0.710-0.894)$ & 61.1 & 93.3 & 97.6 & 40 & 9.92 & 0.36 \\
\hline & & & $>7.84$ & & 93.6 & 40 & 86.6 & 60 & 1.56 & 0.16 \\
\hline \multirow[t]{2}{*}{ M2 } & $2.419(1.444-4.502)$ & 0.001 & $>9.54$ & $0.838(0.737-0.912)$ & 58.1 & 100 & 100 & 36.6 & & \\
\hline & & & $>7.986$ & & 87.1 & 66.7 & 91.5 & 55.6 & 2.61 & 0.19 \\
\hline M3 & $2.039(1.342-3.099)$ & 0.001 & $>8.83$ & $0.827(0.724-0.904)$ & 67.7 & 93.3 & 97.7 & 41.2 & 10.16 & 0.35 \\
\hline M4 & $2.927(1.611-5.318)$ & $<0.0001$ & $>8.57$ & $0.859(0.761-0.928)$ & 61.3 & 100 & 100 & 38.5 & & 0.39 \\
\hline M6 & $2.72(1.573-4.705)$ & $<0.0001$ & $>6.42$ & $0.865(0.767-0.932)$ & 70.8 & 100 & 100 & 45.5 & & 0.29 \\
\hline
\end{tabular}


qSOFA score, SIRS score and established risk models in different cohort and clinical settings of SFTS patients. Our results showed that qSOFA score was the only common index associated with in-hospital mortality of SFTS patients in different settings, and was the only independent risk factor for patients admitted in ICU.

M1 composed of age, AST, qSOFA and SIRS score has the highest predictive value for in-hospital mortality in all patients (Table 2, Fig. 2a) and M5 has the highest predictive power for those of age $\geq 60$ years (Table 3, Fig. $2 \mathrm{~b}, \mathrm{c}$ ) and relative low predictive power in those enrolled in ICU (Table 2). However the modified simpler ones without AST and SIRS scores have higher predictive validity than M1 in these patients. This suggests that different models have diverse predictive values in different clinical settings. And clinical manifestation indices without biochemical parameters can also have high predictive efficacy for prognosis of diverse cohort SFTS patients.

Although model 5 (M5) holds the highest predictive validity for patients at age $\geq 60$ years, the complexity of M5 composition limited its application to initial admission when some parameters are not at hand. Survival analysis illustrated profound differences between low risk and high risk groups of M4 and M5 $(p<0.0001)$. Because of its simpler component parts, M4 can be used as a suitable risk model for predicting prognosis of SFTS patients at age $\geq 60$ years.

SOFA score has been demonstrated the optimal indicator for sepsis in patients enrolled in ICU [5]. Its utility is restricted outside the ICU because many SOFA variables, such as cardiorespiratory, neurologic organ dysfunction are not measured routinely. qSOFA requires only a clinical examination and physiological parameters, including respiratory rate, mental status, and systolic blood pressure, and therefore is readily applicable which is especially valuable in resource-limited settings [7].

Though SIRS score is inferior to qSOFA score for the prediction of adverse outcome in our results, it adds the advantage of predictive value together with age and /or other parameters compared with the simpler qSOFA score. A recent study suggested that at least 2 of qSOFA score could be an alternative parameter for septic patients fulfilled the SIRS criteria [11]. In our results we show that qSOFA score of more than zero can predict adverse outcome of SFTS with high sensitivity which indicates that qSOFA score as a synthetical index can be used combining with age or used solely as a sensitive indicator for predicting prognosis of SFTS.

As a systemic virus infectious disease with overall dysfunction of cellular and humoral immunity [12, 13], SFTS patients were susceptible to bacterial and fungal infection. Procalcitonin (PCT) is a useful parameter to guide antibiotic therapy in severe sepsis patients [14]. While it is not an independent risk factor associated with in-hospital mortality in our results. The reason may be that on one hand it presents as a semiquantitative result, and on the other hand, PCT is not specificity to secondary bacterial infections of virus infection [15]. Other inflammatory parameters such as Hs-CRP and WBC are not independent risk factors for in-hospital mortality except for aged patients which suggest that a single index can not reflect disease status and predict disease outcome.

There are several limitations in this study. First, it is a single center study with a relatively small number of patients which may affect the predictive efficacy of the indices and models. Second, SFTSV infection can influence the number of WBC, therefore impact the accuracy of SIRS score which may influence the predictive power of established models containing SIRS score. Third, the models need further validation in prospective studies including large number of patients.

\section{Conclusions}

In all, risk score models containing qSOFA have high predictive value for in-hospital mortality of SFTS patients with different clinical settings.

\section{Abbreviations}

ALT: Alanine aminotransferase; AST: Aspartate aminotransferase; AUCs: Area under ROC curve; BUN: Blood urea nitrogen; hs-CRP: High-sensitivity Creactive protein; ICU: Intensive care unit; $L R+$ : Positive likelihood ratio; LR: Negative likelihood ratio; NPV: Negative predictive value; PPV: Positive predictive value; PCT: Procalcitonin; qSOFA: Quick sequential organ failure assessment; ROC: Receiver-operating characteristic; RT-PCR: Reverse transcription-polymerase chain reaction; SEN: Sensitivity; SPE: Specificity; SIRS: Systemic inflammatory response syndrome; sCr: Serum creatinine; SFTS: Severe fever with thrombocytopenia syndrome; SFTSV: SFTS virus; WBC: White blood cell

\section{Acknowledgements}

The authors thank all of the contributors in SFTS diagnosis and treatment.

\section{Authors' contributions}

LW and ZZ conceived and designed the research. $\mathrm{KD}$ and $\mathrm{CH}$ collected clinical samples. LW performed the data analysis and modeling. LW wrote the manuscript. All authors reviewed the manuscript. All authors read and approved the final manuscript.

\section{Availability of data and materials}

The datasets used and/or analysed during the current study are available from the corresponding author on reasonable request.

\section{Ethics approval and consent to participate}

This study was conducted according to the Helsinki II Declaration and approved by the ethics committee at the National Institute of Parasitic Diseases, Chinese Center for Disease Control and Prevention. The data used in this study was anonymised before its use.

\section{Consent for publication}

The written informed consent was obtained from every participant in this study.

Competing interests

The authors state that they have no competing interests to declare. 
Received: 2 December 2019 Accepted: 26 July 2020

Published online: 12 August 2020

\section{References}

1. Park SJ, Kim YI, Park A, Kwon HI, Kim EH, Si YJ, et al. Ferret animal model of severe fever with thrombocytopenia syndrome phlebovirus for human lethal infection and pathogenesis. Nat Microbiol. 2019;4:438-46.

2. Li H, Lu QB, Xing B, Zhang SF, Liu K, Du J, et al. Epidemiological and clinical features of laboratory-diagnosed severe fever with thrombocytopenia syndrome in China, 2011-17: a prospective observational study. Lancet Infect Dis. 2018;18:1127-37.

3. Wang L, Zou Z, Hou C, Liu X, Jiang F, Yu H. Score risk model for predicting severe fever with thrombocytopenia syndrome mortality. BMC Infect Dis. 2017; 17:42.

4. Hu LF, Wu T, Wang B, Wei YY, Kong $Q X$, Ye $Y$, et al. The regulation of seventeen inflammatory mediators are associated with patient outcomes in severe fever with thrombocytopenia syndrome. Sci Rep. 2018;8:159.

5. Reece LM, Beasley DW, Milligan GN, Sarathy W, Barrett AD. Current status of severe fever with thrombocytopenia syndrome vaccine development. Curr Opin Virol. 2018;29:72-8.

6. Raith EP, Udy AA, Bailey M, McGloughlin S, Maclsaac C, Bellomo R, et al. Prognostic accuracy of the SOFA score, SIRS criteria, and qSOFA score for in-hospital mortality among adults with suspected infection admitted to the intensive care unit. JAMA. 2017;317:290-300

7. Rudd KE, Seymour CW, Aluisio AR, Augustin ME, Bagenda DS, Beane A, et al. Association of the Quick Sequential (sepsis-related) organ failure assessment (qSOFA) score with excess hospital mortality in adults with suspected infection in low- and middle-income countries. JAMA. 2018;319:2202-11.

8. Schlapbach LJ, Straney L, Bellomo R, MacLaren G, Pilcher D. Prognostic accuracy of age-adapted SOFA, SIRS, PELOD-2, and qSOFA for in-hospital mortality among children with suspected infection admitted to the intensive care unit. Intensive Care Med. 2018;44:179-88.

9. Xiong S, Zhang W, Li M, Xiong Y, Li M, Wang H, et al. A simple and practical score model for predicting the mortality of severe fever with thrombocytopenia syndrome patients. Medicine (Baltimore). 2016;95(52): e5708.

10. Singer M, Deutschman CS, Seymour CW, Shankar-Hari M, Annane D, Bauer $M$, et al. The third international consensus definitions for sepsis and septic shock (Sepsis-3). JAMA. 2016;315:801-10.

11. Abdullah SMOB, Grand J, Sijapati A, Puri PR, Nielsen FE. qSOFA is a useful prognostic factor for 30-day mortality in infected patients fulfilling the SIRS criteria for sepsis. Am J Emerg Med. 2019;21:S0735.

12. Song P, Zheng N, Liu Y, Tian C, Wu X, Ma X, et al. Deficient humoral responses and disrupted B-cell immunity are associated with fatal SFTSV infection. Nat Commun. 2018;9:3328.

13. Li MM, Zhang WJ, Liu J, Li MY, Zhang YF, Xiong Y, et al. Dynamic changes in the immunological characteristics of T lymphocytes in surviving patients with severe fever with thrombocytopenia syndrome (SFTS). Int J Infect Dis. 2018;70:72-80

14. Prkno A, Wacker C, Brunkhorst FM, Schlattmann P. Procalcitonin-guided therapy in intensive care unit patients with severe sepsis and septic shock-a systematic review and meta-analysis. Crit Care. 2013;17:R291.

15. Wu MH, Lin CC, Huang SL, Shih HM, Wang CC, Lee CC, et al. Can procalcitonin tests aid in identifying bacterial infections associated with influenza pneumonia? A systematic review and meta-analysis. Influenza Other Respir Viruses. 2013;7:349-55.

\section{Publisher's Note}

Springer Nature remains neutral with regard to jurisdictional claims in published maps and institutional affiliations.

Ready to submit your research? Choose BMC and benefit from:

- fast, convenient online submission

- thorough peer review by experienced researchers in your field

- rapid publication on acceptance

- support for research data, including large and complex data types

- gold Open Access which fosters wider collaboration and increased citations

- maximum visibility for your research: over $100 \mathrm{M}$ website views per year

At BMC, research is always in progress.

Learn more biomedcentral.com/submissions 